\title{
Anabases
}

ANABASES Traditions et réceptions de l'Antiquité

$8 \mid 2008$

Varia

\section{Antiquité et métamorphose à la Renaissance. Pic de la Mirandole et Giordano Bruno}

Karine Safa

\section{(2) OpenEdition}

\section{Journals}

Édition électronique

URL : http://journals.openedition.org/anabases/152

DOI : 10.4000/anabases. 152

ISSN : 2256-9421

Éditeur

E.R.A.S.M.E.

Édition imprimée

Date de publication : 1 octobre 2008

Pagination : 127-136

ISSN : 1774-4296

\section{Référence électronique}

Karine Safa, "Antiquité et métamorphose à la Renaissance. Pic de la Mirandole et Giordano Bruno », Anabases [En ligne], 8| 2008, mis en ligne le 01 juillet 2011, consulté le 20 octobre 2019. URL : http:// journals.openedition.org/anabases/152 ; DOI : 10.4000/anabases.152 
Anabases 8 (2008), p. 127-136.

\title{
Antiquité et métamorphose à la Renaissance. Pic de la Mirandole et Giordano Bruno
}

KARINE SAFA

\begin{abstract}
Aborder une période aussi COMPLEXe ET Foisonnante que la Renaissance, c'est tenter de la saisir dans ce qu'elle a de spécifique. Il faut tout d'abord reconnaître qu'elle représente une véritable rupture avec le Moyen Âge. Une rupture créatrice. Car si la Renaissance rompt avec le Moyen Âge et avec la lecture scolaire de la tradition grécolatine, c'est pour instaurer une autre relation et retrouver l'Antiquité selon ses propres vecteurs. En effet, lasse de ce Moyen Âge savant où le discours ne s'exerce plus qu'à travers un discours préalable donnant lieu à une prolifération de commentaires, la Renaissance court-circuite la chaîne des gloses et remonte au référent scripturaire de base. Elle tente alors une reprise du passé au sens proprement kierkegaardien du terme ${ }^{1}$. Le passé régénère le présent et l'éclaire. Il ne s'agit pas de réduire les traditions les unes aux autres ou de les concilier mollement en effaçant leurs différences. La grandeur de l'humanisme, c'est au contraire de composer les contraires et de permettre l'interférence des traditions pour mieux entendre ce qu'elles ont à nous dire. C'est la nymphe Écho qui renvoie et multiplie la voix humaine à l'Infini alors que Narcisse, en s'aimant lui-même, se fige et va vers sa mort.
\end{abstract}

1 La répétition est tout sauf répétition du même, chez Kierkegaard. Loin de faire la synthèse des différences et des contradictions, la "seconde immédiateté ", comme il la nomme, maintient la tension générée par les différences. C'est alors seulement que le passé remémoré peut s'enrichir et générer de nouveaux objets ou de nouvelles formes de pensées. 


\section{L'humanisme de la Renaissance}

\section{Une apologétique de la diversité}

Cette « apologétique de la diversité » dont parle Georges Gusdorf ${ }^{2}$ a pour vocation de fonder un humanisme anti-dogmatique. Conscient désormais de faire partie d'une histoire, l'homme de la Renaissance sait qu'il n'est une voix de trop (sagesses révélées, orphisme, hermétisme, aristotélisme, platonisme, etc.) pour célébrer le miracle humain et rendre hommage à la vérité. La vérité se dit désormais sous de multiples formes : la culture de la différence devient exigence primordiale et les sagesses antiques sont le terrain privilégié de cette empathie. Quand Pic de la Mirandole, dans le De Ente et Uno, montre la nécessité de saisir les deux grands principes métaphysiques ensemble et non dans leur antagonisme, il fait preuve d'un humanisme authentique. En effet, pour lui, il ne s'agit pas de choisir l'un au détriment de l'autre. Tous les deux ont leur nécessité propre puisqu'ils ne rendent pas compte d'une même réalité : le premier témoigne d'un Absolu insaisissable et solitaire alors que le deuxième nous introduit dans une logique de la relation et de la participation. Les deux principes sont nécessaires pour rendre compte de la nature des choses. La pensée est donc stratégiquement maintenue dans une saine tension, ici entre l'Un et l'Etre, sans que jamais ils ne soient confondus. La Renaissance fait ainsi apparaitre une philosophie du dilemme ou de la disjonction. Les aspérités, les contradictions auxquelles la pensée est sans cesse confrontée, ont quelque chose de salutaire.

Nicolas de Cues qui préfigure la Renaissance ${ }^{3}$ nous en offre une belle image avec son expérience orientale. Parti à Constantinople pour chercher un accord avec l'Église d'Orient, il revient en apparence bredouille. Cependant, sur le chemin du retour, il est saisi d'une vision fulgurante. Lui qui croyait pouvoir ramener les orthodoxes dans le giron de Rome, il réalise ce que Byzance lui a apporté et comprend que la vérité n’est pas dans la soumission d'une tradition à l'autre mais dans leur unité. Désormais, il sait qu'il faut regarder Rome à travers Byzance (et inversement), chercher dans l'autre ce même que l'on connaît mal (je est déjà un autre), retrouver l'unité dans la multiplicité,

2 Les sciences humaines et la pensée occidentale, 5, Dieu, la nature, l'homme au siècle des Lumières, Paris, Payot, 1972.

3 C'est avec Nicolas de Cues qu'apparaît pour la première fois la thèse d'un univers infini fragilisant les facultés cognitives de l'homme. C'est d'ailleurs dans ce passage d'un "monde clos à un univers infini " (titre d'un ouvrage d'Alexandre Koyré) qu'on peut situer le début d'une première Renaissance : celle du Quattrocento (il y a une Renaissance plus tardive, propre au Concile de Trente et à la vague luthérienne qui ne fait pas l'objet de cette contribution). 
l'Infini dans le fini. Il faut devenir un voyant et réaliser l'expérience jubilatoire de la coïncidence des opposés ${ }^{4}$.

Pour l'humanisme, on le voit, il n'est d'antagonisme qui ne soit fraternel, il n'est de communion hâtive qui ne devienne suspecte.

\section{Une période de crises}

Toutefois, que de crises la Renaissance a-t-elle dû traverser avant d'offrir à la postérité le visage lumineux de l'humanisme. Monde en perpétuel changement, découvertes géographiques et scientifiques, tout concourt à la Renaissance à déstabiliser l'homme et à lui faire perdre ses repères. Ne croyait-il pas, comme les Anciens, être le centre du monde dont il tirait maints titres de gloire? Et voilà que la formidable révolution copernicienne vient le décentrer, l'expulser de sa position confortable pour lui apprendre la nouvelle catastrophique d'un univers infini. Ce bouleversement cosmologique entraîne une véritable révolution spirituelle. Destitué d'une position éminente, l'homme doit désormais trouver une réponse à la disproportion nouvelle qui s'impose entre lui et le monde. Comment continuer à jouer ce rôle rassembleur dans un " univers dont le centre est partout et la circonférence nulle part ?" Cette tension, ce nœud sont au cœur du "drame " renaissant. Moment unique de l'histoire de la pensée où l'homme se libère des carcans du passé pour mieux découvrir sa servitude. Et pourtant, c'est au creux de cette déchirure, de cette contradiction que l'homme de la Renaissance va se construire un destin.

\section{La mythologie comme fondement}

L'antiquité, c'est aussi pour nos renaissants ce vivier de forces obscures où l'irrationnel et l'imaginaire sont autant constitutifs de l'homme que sa raison ${ }^{5}$. L'abstraction du

4 Présentée comme une révélation, l'idée de la coïncidence des opposés construit une logique non duelle et libère l'intelligence du principe de contradiction aristotélicien. Il faut être capable de voir de l'œil même de Dieu, c'est-à-dire sur le mode de l'unité. Car dans l'unité de la vision divine toute diversité est surmontée. La théorie de la docte ignorance, qui est une réflexion critique au sens kantien du terme sur les limites de la connaissance, se fonde sur le constat d'une disproportion irréductible entre le fini et l'infini. Dès lors, pour rejoindre l'infini, il faut tenter l'expérience du " passage à la limite ». Les spéculations mathématiques, sur lesquelles Nicolas de Cues s'appuie, aident à comprendre comment à l'infini les opposés coïncident comme le droit et le courbe, le point et la ligne, etc. Dans ce passage à la limite, les murs tombent et les affirmations contraires trouvent un lieu de conciliation ou de réconciliation.

5 Jean-François Pic de la Mirandole, neveu de Jean Pic, publie l'un des premiers traités sur le sujet, De l'imagination, et montre le rôle nécessaire de cette faculté dans les opérations sensibles et intellectuelles. 
concept n'impose jamais sa tyrannie aux fulgurances de l'expression symbolique. Selon Bruno Pinchard, fervent interprète de la Renaissance, "la philosophie a trop servi jusqu' ici à justifier une séparation croissante entre le calcul rationnel et les jeux de l'imaginaire. Notre but est au contraire de replacer l'intention philosophique dans ses sources mythologiques et symboliques. Ainsi la philosophie demeurera-t-elle notre dernier lien avec l'âge des dieux. La philosophie ne peut plus assumer ses fonctions dans l'histoire de la culture en se limitant [...] à une simple logique du concept. Un concept n'a une vocation historique, c'est-à-dire humaine, que s'il procède d'un imaginaire et qu'il se constitue comme la force d'intelligibilité de cet imaginaire ${ }^{6}$."

Il nous faut imaginer le philosophe de la Renaissance comme un archéologue de la pensée qui cherche à reconstituer l'édifice humain à travers la moindre strate. Ce faisant, loin de rencontrer un amas de poussière, il fait une expérience spirituelle. Comme l'a très bien vu André Malraux, la Renaissance ne fait pas un inventaire du passé, pas plus qu'elle ne réalise un reportage sur les dieux de l'Olympe, car il s'agit bien d'abord d'expérience et non de représentation ${ }^{7}$. Le génie italien a le courage de sortir de la rigidité du concept, et l'héroïsme de reconquérir son héritage à travers une disposition au changement.

Il n'est jusqu'à son attachement instinctif aux figures allégoriques pour exprimer la force rédemptrice de l'irrationnel. Car enfin dans cet univers de fables et d'errance, l'homme de la Renaissance voit le théâtre de sa condition. Il perçoit dans les figures de la mythologie les mouvements de son être, d'un être qui, à l'image des esclaves de Michel Ange, secoue la gangue de la matière pour exorciser sa finitude. Il croit reconnaître dans cette gigantesque fresque de vicissitudes, de peine et de douleur un effort héroïque pour fixer l'être dans l'éternité. Les héros de la mythologie sont autant de compagnons d'infortune, si l'on peut dire, pour qui le temps offre l'image insupportable de la dégradation.

À travers ces formes sans cesse mouvantes, qui ne s'arrachent à une figure que pour en épouser une autre, on peut voir l'expression du changement universel mais ce qu'il faut surtout saisir dans la fluidité des figures mythologiques c'est le rêve de perdurer. Etre ivre de possibles, c'est pouvoir ne jamais mourir. Projet grandiose! Ovide ne parle-t-il pas de ces malheureux qui «s'affranchirent par la mort de la peur de mourir " ? (Mét. III) et Myrrha, quand elle supplie les dieux de faire d'elle " un autre être à qui soient interdites et la vie et la mort " (Mét. X) ne fait que nous dire la plus grande crainte qui ait hanté l'humanité. Médée, quant à elle, détient le secret du philtre de jouvence qu'elle laisse, avec jubilation, « écouler dans la gorge » de Eson le vieillard.

6 Article de présentation sur Internet du centre d'études supérieures sur la Renaissance de Tours dont Bruno Pinchard fut le directeur. URL : www.cesr-tours.fr.

7 André Malraux, L'Irréel, La Métamorphose des dieux, Paris, Gallimard, 1983, p. 115. 
Exaspéré par ses limites, hanté par sa finitude, l'homme de la Renaissance voit dans la métamorphose comme une brèche pour entrer dans la voie royale mais étroite de l'immortalité.

\section{La métamorphose à la Renaissance}

Deux œuvres sont particulièrement éclairantes sur la fonction et le rôle joués par ce philosophème : celles de Giordano Bruno et de Pic de la Mirandole.

Giordano Bruno, en homme de son temps, se situe dans le cadre d'une cosmologie infinitiste. Il en tire la conséquence d'une continuité de toute matière vivante. Le monde est en perpétuel changement, les êtres en perpétuelle transformation. La uarietas s'impose ici comme le principe même de la vie organique et psychique. Dès lors, la loi des vicissitudes qui régit le monde et par laquelle rien n'est essentiellement perdu fait de la mort un simple passage, le passage d'une forme d'être à une autre : "c'est ce que Pythagore a été capable de comprendre, lui qui ne craint pas la mort, mais l'attend comme une transformation ${ }^{8}$ ".

Intégrer la roue des métamorphoses, c'est assurer la continuité de la vie dans un perpétuel dépassement de soi. Tel est le destin du furieux héroïque qui poursuit l'Absolu avec passion jusqu'à la perte de soi ${ }^{9}$. C'est ce que donne à voir le mythe d'Actéon réinterprété par Bruno.

On connaît plus ou moins la malheureuse partie de chasse du petit-fils d'Apollon. Pour Bruno, le mythe donne à voir avant tout une chasse héroïque de la sagesse.

Actéon, accompagné de sa meute, poursuit son gibier, un cerf (la Sagesse). Au cours de la partie de chasse, il surprend Diane (la Nature) nue, dans son bain. Furieuse, la déesse veut lui infliger un terrible châtiment. Elle le transforme alors en Cerf (l'objet de sa quête) et excite sa propre meute contre lui. Destin apparemment funeste, le Cerf Royal finit par mourir sous les crocs des chiens déchaînés. Pourtant, cette mort est une mort glorieuse, puisqu'elle permet à Actéon de s'unir à la Nature et de rentrer dans la communion de Dieu.

La fureur héroïque aura servi de formidable moteur pour percer le mystère d'un dieu dissimulé dans une nature qui en est l'âme, le Verbe ou encore la chair. Dans cette étreinte amoureuse qui célèbre des noces bien singulières, la nuit de la finitude semble se dissiper.

8 De la cause, du principe et de l'un, dial. V, in CEuvres Complètes, III, Paris, Les Belles Lettres, 1996.

9 L'éternité, chez Bruno, n'est en aucune façon promesse d'immortalité pour l'âme individuelle mais participation au cycle des transformations du monde. C'est pourquoi l'œuvre de Bruno a-t-elle pu être considérée comme anti-humaniste. Elle tend, en effet, à dissoudre la singularité dans une conception fusionnelle avec la nature. 
À ceci près que la religion philosophique que propose Bruno est une religion de stricte immanence. La question que l'on peut dès lors se poser face à ce final sacrificiel est de savoir si le sage peut échapper à la roue des métamorphoses quand il passe en Dieu ? Ne reste-t-il pas au contraire prisonnier de la loi des vicissitudes, de cette loi universelle qui semble être Dieu lui-même caché au plus profond de la Nature?

Toute autre est la vision de Pic. Alors que chez Bruno, on l'a vu, la métamorphose est une traduction païenne et naturaliste de la résurrection chrétienne, pour Pic de la Mirandole, elle est promesse de déification dans l'au-delà. Le traitement qu'il réserve au schème du microcosme, sa manière de reprendre cette thématique chère aux Anciens comme aux auteurs du Moyen Âge tout en la dépassant est révélatrice d'un humanisme profondément christologique.

\section{Signification du microcosme : vers une métaphysique de l'esprit}

Le schème du microcosme met l'accent sur la nature infiniment accueillante et flexible de l'homme en tant que petit-univers. Créature du milieu, participant des deux natures, sensibles et intelligibles, c'est en lui que les êtres confluent et prennent sens. "L'homme a quelque chose de toute créature, nous dit Pic : il a en effet l'être en commun avec les pierres, la vie en commun avec les arbres, le sentir en commun avec les animaux, l'intelligence en commun avec les anges; si donc l'homme a quelque chose en commun avec toute créature, selon quelque chose l'homme est toute créature ${ }^{10}$. " Copule du monde, lien entre les êtres, l'homme a le privilège d'être un médiateur universel ${ }^{11}$. Il assume la totalité des êtres et, ce faisant, révèle une nature métamorphique.

C'est le rapport de l'homme à la nature qui éclaire cette vocation. En effet, la nature est une réserve inépuisable de forces, une matière infinie où les germes de l'esprit sommeillent.

Cette métaphysique, que l'on peut qualifier de métaphysique de l'esprit, établit une continuité sans faille entre l'esprit et la nature. Elle s'enracine résolument dans le néoplatonisme antique. On connait la fameuse triade plotinienne Être-Vie-Pensée. Lêtre et la pensée sont si étroitement conjoints à travers la vie que Plotin se demande si le sommet de la vie intellectuelle n'est pas le sommet de la vie elle-même. Les formes ne viennent réellement à la vie que parce qu'elles sont contemplées par l'intelligence mais l'intelligence n'est expressive que si elle est nourrie par l'ordre du créé ${ }^{12}$.

L'Heptaplus, 2e exposition, Paris, Arma artis, 1978.

Sans doute n'est-ce pas en vain que Nicolas de Cues surnommait l'homme « ce second Dieu " avec les résonances christiques que l'expression implique.

Pour Plotin, il y a une parenté et une continuité parfaite entre la plus humble des créatures et la plus élevée. On peut lire dans un texte émouvant de la III ème Ennéade : «Si l'objet contemplé par l'intelligence doit vivre, ce n'est plus de la vie d'une plante, d'un animal 
La Renaissance, en reprenant à son compte la métaphysique de l'esprit, constitue le point d'orgue d'un vaste mouvement dans l'histoire de la philosophie, celui qui répond au grand problème métaphysique de l'accord entre Pensée et Réel par la réversibilité des processus physiques et mentaux. Toutefois, c'est avec héroïsme que la Renaissance célèbre ce fascinant jeu de miroir entre nature et esprit. Héroïsme car la métaphysique de l'esprit se développe dans un contexte très particulier, celui d'une crise aiguë du centre conjuguée, comme nous le verrons, à une liberté qui se manifeste dans sa double puissance olympienne et titanesque.

La puissance cosmique de l'homme se fonde donc sur cette métaphysique: l'homme ne mérite le nom d'homme que s'il est capable d'exprimer le monde et l'âme individuelle ne peut s'accomplir qu'en étant reliée à l'âme du monde. C'est pourquoi la Renaissance est-elle l'âge du lien par excellence, fidèle en cela au néoplatonisme chrétien, contrairement à l'âge classique qui met à distance une nature devenue suspecte ${ }^{13}$.

Cependant, le motif de microcosme n'est jamais considéré comme une fin en soi chez Pic de la Mirandole. Il ne saurait non plus à lui seul conférer à l'homme sa dignité. Les métamorphoses "mondaines " de l'homme ne sont que prolégomènes à une métamorphose divine; et la capacité de résumer le monde n'intéresse Pic de la Mirandole que dans la mesure où elle révèle la liberté créatrice de l'homme. Liberté qui se fonde sur la qualité d'imago dei.

\section{Image du monde, Image de Dieu}

On connaît les pages somptueuses du fameux Discours lors du récit de la Création. En créant le monde, le Créateur donne à chaque être une forme spécifique. Quand il parvient à l'homme, l'architecte tout-puissant semble avoir comme une hésitation, une défaillance. Le Créateur ayant donc épuisé nombre d'idées et d'archétypes, «il décida qu'à celui à qui il ne pouvait rien donner en propre serait commun tout ce qui avait été le propre de chaque créature [...]. Il prit donc l'homme, cette œuvre à l'image indistincte, et lui parla ainsi : "Je ne t’ai donné ni place déterminée, ni visage propre, ni don particulier afin que ta place, ton visage et tes dons, tu les veuilles, les conquières et les

ou d'aucun être animé. Sans doute ces vies sont des pensées de différentes espèces; il y a une pensée de la plante, une pensée de l'animal doué de sens, une pensée de l'être doué d'une âme ; comment des pensées ? Oui, puisque ce sont des raisons ; toute vie est une pensée, mais une pensée plus ou moins obscure comme la pensée elle-même ". Enn, III, 8, 8, Paris, Les Belles Lettres, 1963, p. 162-163.

13 Il faut toutefois préciser que les germes de la philosophie classique se trouvent déjà à la Renaissance. Il suffit de se pencher sur la signification métaphysique de la perspective dans l'art. Cette vision traversante n'est-elle pas expérience de déracinement pour l'esprit? Expérience de déracinement car, à travers la recherche d'un point fixe, c'est le sujet pensant, ou la représentation du sujet pensant, plus exactement, qui devient le principal repère. 
possèdes par toi-même [...] Je ne t'ai fait ni céleste ni terrestre, ni mortel ni immortel, afin que, souverain de toi-même, tu achèves ta propre forme librement, à la façon d'un peintre ou d'un sculpteur..." 14 ".

L'homme est ainsi la seule créature dans l'ordre du créé à pouvoir se donner sa propre forme. Sans image, indéterminé, il devient libre créateur de lui-même. Idée révolutionnaire pour l'époque, il faut désormais admettre qu'il n'a de nature que celle qu'il s'octroie lui-même.

Nous voyons ce que ce pouvoir a de pervers et d'inquiétant. Si la Renaissance a glorifié plus que toute autre époque la liberté humaine, elle est aussi à l'origine des tentations de dérives faustiennes de cette liberté. Certains commentateurs ont d'ailleurs considéré un peu trop hâtivement que cette liberté infinie était, chez Pic de la Mirandole, une puissance prométhéenne permettant à l'homme d'égaler Dieu en ses pouvoirs.

Il est en tout cas une expression, dans l'œuvre du Florentin, à laquelle il faut être attentif : celle de vis titanica ${ }^{15}$. La puissance métamorphique semblerait double : une force titanique qui est puissance de dispersion, rupture avec l'Un et perte dans le multiple ; et une force olympienne, figurée dans l'œuvre par l'échelle de Jacob, qui est force de rassemblement.

Sans être forcément un moment négatif, la vis titanica pourrait signifier la part mythologique de l'homme, sa face obscure. Elle serait aussi l'expression du décentrement du monde, de son irréductible multiplicité.

Quoi qu'il en soit, et quel que soit le type de métamorphose auquel l'homme est confronté, il s'agit bien moins pour lui de changer de nature (nous sommes loin des théories de la métempsychose) que d'approfondir la sienne comprise comme infinie et donc comme le fruit d'une perpétuelle conquête. Il n'est de perfection pour l'homme qu'à l'intérieur de sa nature d'homme, comme se plaît à le répéter le Cardinal de Cues.

Sa liberté n'en reste pas moins un redoutable pouvoir qui met l'accent sur la responsabilité de l'homme. Il lui appartient d'achever la Création en la ramenant vers l'intelligence divine ou, au contraire, de précipiter sa chute vers le chaos. Ce qui fonde la dignité de cet homme sans qualités est aussi ce qui peut l'asseoir dans la misère. "Il peut tout bouleverser et détruire, comme il peut tout rédimer dans une transfiguration libératrice ", nous dit Eugenio Garin ${ }^{16}$.

14 Discours sur la dignité de l'homme, Paris, Éditions de l'Éclat, 1993, p. 6-7.

15 "Pénétrant toute chose du centre au centre, tantôt nous descendrons, déchirant comme Osiris l'un en multiple, avec la force d'un titan, tantôt nous monterons, rassemblant comme les membres d'Osiris, le multiple en Un." (p.21) Cette expression tirée des premières pages du discours a fait couler beaucoup d'encre. Certains y ont vu la puissance démoniaque de l'homme livré à lui-même. 
En réalité, il y a bien moins antagonisme entre les deux entités image du mondeimage de Dieu que complétude ${ }^{17}$. Si Pic de la Mirandole insiste tant sur les capacités métamorphiques de l'homme qui n'a aucune forme en propre car il peut les devenir toutes, c'est qu'elles préparent cette démesure ultime qu'est le passage en Dieu ${ }^{18}$. Comme le précise Pierre Magnard, le moment de l'invocation théologique est essentiel. C'est alors seulement que " la vérité est sauve dans l'unité de la pensée et de l'être, alors aussi la diversité humaine devient unanimité ${ }^{19}$ ". Pour Pic de la Mirandole, sans une assistance divine, les variations humaines sont dispersion ; sans le secours d'une Main Puissante qui hisse et rassemble, notre nature « multiforme et changeante " est vouée à l'errance.

La métamorphose fonctionne comme si elle cherchait à se substituer aux transcendantaux traditionnels que sont l'Un et l'Être : face à la crise du centre et à l'esprit qui éprouve douloureusement l'inadéquation de la pensée à la réalité, ce philosophème tente d'opérer comme un principe de restauration.

Si l'enjeu de la métamorphose est bel est bien la déification de l'homme, la question qui se pose toutefois est la dignité philosophique que l'on peut accorder à un concept qui implique le passage, le changement. Toute la difficulté, pour le philosophe, est de tenir compte de cette réalité fondamentale qui traverse la Renaissance, en constitue même l'esprit, avec ses ramifications mythologiques profondes, tout en s'interrogeant sur la structure qui peut permettre de la penser. Il est en tout cas difficile d'accorder à la métamorphose le statut de transcendantal auquel elle aspire, puisque le transcendantal, par nature, en tant que modalité de l'Être, ne peut être affecté par le changement, au risque de devenir un principe physique plutôt que métaphysique. Si la Renaissance est héroïque, le philosophe qui réfléchit sur la Renaissance doit faire preuve d'un même héroïsme, car il lui incombe de penser l'impensable, un concept qui introduit une discontinuité dans l'acte mental. L'héroïsme consiste à voir le problème en face plutôt que d'effacer hâtivement la spécificité irréductible de la Renaissance et de se contenter de la lire dans une continuité un peu trop fade avec le Moyen Âge.

17 La qualité d'imago dei situe la pensée dans un double mouvement de développement (explicatio) et d'enveloppement (complicatio) pour reprendre les termes de Nicolas de Cues. Le développement implique que Dieu est présent dans chaque créature, immanent à toute chose et l'enveloppement caractérise le mouvement de rassemblement. C'est à ce double mouvement qu'est invitée la Mens Humana en tant qu'image de la pensée divine. La pensée s'enracine d'abord dans le sensible - c'est par son entremise que les êtres participent à la pensée divine - pour remonter ensuite de la multiplicité des choses vers le principe.

18 Cf. notre ouvrage L'humanisme de Pic de la Mirandole, l'esprit en gloire de métamorphoses, Paris, Vrin, 2001.

19 "Le singulier universel", in Fine Follie ou la catastrophe humaniste, Paris, Honoré Champion, 1995, p. 237. 


\section{Conclusion}

La catégorie capable de rendre compte de la métamorphose reste donc à imaginer. Il faudrait faire en sorte que la métamorphose soit plus que la face rêveuse du concept ou un concept en souffrance, si l'on peut s'exprimer ainsi.

Cette question est terriblement contemporaine; elle nous parle à plus d'un titre. La métamorphose, on l'a vu, traduit cet effort héroïque qui traverse l'histoire pour trouver une réponse au problème de la finitude. De plus, elle engage un humanisme très particulier qui peut servir de garde-fou aux tentations absolutistes dont regorge l'histoire contemporaine. C'est en effet un philosophème qui semble taillé sur mesure pour les temps de crise.

Apologétique de la diversité et humanisation vont de pair. La sagesse du lézard, figure si chère à Pic de la Mirandole, consiste à rencontrer sa singularité au plus profond de l'altérité de telle sorte qu'il n'est de vraie singularité, de véritable humanisme qui ne soit universel ${ }^{20}$. La force du singulier c'est qu'il récapitule l'espèce; la pauvreté de l'individualité c'est qu'elle repose sur soi. "Il y a une grande rareté de l'homme en cette saison ", disait déjà Montaigne. Il n'est d'humanisme véritable qui ne soit assomption de l'universel dans le singulier. C'est cette rencontre que la métamorphose met en scène. Revêtir un maximum de formes, c'est se singulariser et donc s'humaniser davantage.

La Renaissance fécondée par l'Antique et nourrie par la culture judéo-chrétienne nous ouvre la voie très actuelle de cet humanisme différentiel et dialectique qui tente de mettre à distance la tentation du nihilisme propre aux temps modernes. Si la plongée dans la durée n'est que disgrâce et déclin, la puissance de métamorphose reste pour l'homme cette espérance de ressembler au divin dans sa gloire pérenne.

Karine SAFA

Docteur en philosophie

Écrivain, chercheur

10, rue de Montevideo

75116 Paris

karinesafa@yahoo.fr

20 Cf. l'excellent livre du grand spécialiste de la philosophie de la Renaissance, Pierre Magnard, Questions à l'humanisme, Paris, PUF, 2000. 